Les sources de l'Histoire des Mines : Nouveaux outils, Nouvelles approches

\title{
Le rail et la route
}

Dominique Larroque

\section{(2) OpenEdition}

1 Journals

Édition électronique

URL : http://journals.openedition.org/dht/842

DOI : $10.4000 /$ dht. 842

ISSN : 1775-4194

Éditeur :

Centre d'histoire des techniques et de l'environnement du Cnam (CDHTE-Cnam), Société des élèves du CDHTE-Cnam

\section{Édition imprimée}

Date de publication : 1 décembre 2008

Pagination : 198-200

ISBN : 978-2-95-30779-2-6

ISSN : 0417-8726

\section{Référence électronique}

Dominique Larroque, «Le rail et la route », Documents pour l'histoire des techniques [En ligne], 16 | 2e semestre 2008, mis en ligne le 08 octobre 2010, consulté le 24 septembre 2020. URL : http:// journals.openedition.org/dht/842 ; DOI : https://doi.org/10.4000/dht.842

Ce document a été généré automatiquement le 24 septembre 2020.

(c) Tous droits réservés 


\title{
Le rail et la route
}

\author{
Dominique Larroque
}

\section{RÉFÉRENCE}

Paul Wohl et A. Albitreccia, La route et le rail dans quarante pays, Les documents de la Chambre de commerce internationale, Tours, Arrault et cie, 1934, 499 p. et tableaux Marcel De Conink, La mort du rail. La renaissance économique par l'automobile, Les Cahiers bleus, 1931, $121 \mathrm{p}$.

André Guillerme dir., Chroniques routières. L'âge du pétrole, Paris, ministère de l'écologie, du développement et de l'aménagement durable, 2007, 274 p. et DVD-ROM

1 Durant l'Entre-deux-guerres, accompagnant la montée en puissance de l'industrie automobile, commence le débat sur la concurrence entre le rail et la route. Le ton fut souvent polémique. "Chaque mode de transport a la part d'existence que lui confère non pas ses mérites passés (les chemins de fer), mais son mérite actuel (l'automobile); il faut laisser agir la loi inéluctable de la sélection naturelle » soutient le baron Pétiet, alors Président de la Fédération nationale de l'automobile. Un peu trop commode ce credo libéral, lui rétorquent les partisans du chemin de fer, «quand on sait que vos poids lourds et vos voitures de tourisme roulent sur une chaussée entretenue, balisée et sécurisée aux frais de l'État et donc du contribuable ! ». De plus, le rail est soumis à des contraintes réglementaires de service (fréquences, tarifs, horaires, etc.) qui lui interdisent de réagir au défi de son concurrent en ajustant ses propres prestations. La bibliothèque du CDHTE vient de s'enrichir de deux ouvrages contemporains traitant de cette relation difficile « rail-route » ${ }^{1}$. L'un est la publication d'un rapport établi en 1934 par Paul Wohl et A. Albitreccia pour la Chambre de commerce internationale : La route et le Rail dans quarante pays, l'autre est un petit essai polémique signé Marcel de Conink: La mort du rail. La renaissance économique par l'automobile, 1931.

2 Le premier ouvrage, 499 pages, constitue une mine de renseignements. Les 40 pays ont été choisis dans les cinq continents. Pour le continent américain: les États-Unis, le Canada, le Mexique, les «États-Unis du Brésil ». L’Europe, avec 19 pays, est la mieux 
représentée. Pour les contrées plus lointaines, les auteurs ont retenu l'Inde, les Indes néerlandaises, l'Indochine française, la Chine, le Japon. Pour l'Asie " antérieure » : les chemins de fer syriens et persans, les seuls dont la documentation soit accessible, précisent les auteurs. Un grand absent : la "nouvelle Russie ", si ce ne sont quelques indications succinctes. L'ouvrage est divisé en deux grandes parties. La première rassemble les données juridiques et statistiques du rail et de la route par pays. La seconde propose une analyse transversale autour de quatre grands thèmes: les bases juridiques des concessions et des licences d'exploitation; les charges fiscales; les mesures prises par les chemins de fer face à la concurrence; la législation sociale et ouvrière. Chacun d'eux est divisé en deux chapitres : l'un, où les deux auteurs tentent de dégager des principes généraux ; l'autre destinée à souligner les multiples variantes nationales. L'ouvrage s'achève par trois tableaux comparatifs synoptiques ventilant les données par pays. Rien n'est plus fructueux qu'une confrontation internationale de cette nature pour évaluer le positionnement d'un pays vis à vis des enjeux de cette époque. On apprend ainsi que la France des chemins de fer sur l'échelle de l'interventionnisme étatique est en retrait par rapport à l'Italie, le Japon, les États baltes, la Yougoslavie, la Hongrie, la Pologne, l'Australie etc., où la majeure partie du réseau est propriété des États. Dans tous les pays le rail est considéré dès l'origine comme un secteur hors norme, d'intérêt général, et à ce titre son exploitation fut très encadrée, y compris dans la très libérale Amérique du Nord. Concernant la route, la France est déjà en bonne position. Elle est en tête du peloton au regard de la densité du réseau : $120 \mathrm{~km}$ pour $100 \mathrm{~km}^{2}$; 95 en Grande Bretagne ; 62 aux USA, et 45 seulement en Allemagne. C'est une vieille histoire qui remonte à la «création de l'École des Ponts et chaussées, dès le règne de Louis XV » soulignent les auteurs. En nombre d'automobiles en circulation en revanche, les USA laissent loin derrière eux les autres pays : $74 \%$ des automobiles recensées dans le monde en 1931, soit près de 26 millions de véhicules. Outre-Atlantique, la voiture "jouit d'un prestige incomparable», les États membres comme le gouvernement fédéral hésitent à lui imposer des règles susceptibles de retenir la "main invisible» du marché. Au Japon en revanche, le trafic automobile à vocation commerciale (voyageurs et marchandises) se voit placé sous la tutelle de son concurrent : toute création est soumise au contrôle de l'instance ferroviaire suprême, le «Railway Department». Au fil de la lecture, on append encore qu'en Indochine française, les « populations indigènes » ont manifesté « un goût très vif pour le nouveau mode de transport qui permettait de se déplacer facilement à travers les rizières et facilitait les relations avec les marchés»; "de nombreuses petites entreprises de transport ont été créées par des entrepreneurs peu fortunés », ... « utilisant un matériel vieilli et peu commode ", etc.

3 L'ouvrage de Marcel de Conink, au contraire, abonde en partis pris. Son titre annonce la couleur : en quinze points il démonte, un par un, tous les arguments généralement invoqués en faveur des chemins de fer. Entre le rail et la route, il n'y a pas de " collaboration possible ». Une seule solution s'impose : «Liquider les chemins de fer ", " déferrer les voies » sur tout le territoire et installer à leur place « un réseau national d'auto-routes ». Les tortillards départementaux ne seront pas non plus épargnés: certains d'entre eux pourront être transformés en pistes cyclables ou chemins de promenade. Le rail « condamné à mort » sur les grands réseaux, doit-il survivre en ville ou dans les banlieues? «Pas d'avantage!». Ne parlons même pas du tramway, «que personne ne peut soutenir sérieusement, c'est l'un des fléaux de la circulation urbaine»; parlons du "métro électrique». Il a certes quelque avantage, doit 
péniblement concéder l'auteur, mais à quel prix : escamotage des charges financières, atmosphère souterraine empestée, compression excessive des usagers, fatigue due aux marches interminables dans les couloirs et aux correspondances! Avec des autobus modernes, confortables, à haute fréquence, on aura tôt fait de ramener les malheureux usagers à la surface. Ouvrage polémique donc, mais semble-t-il bien dans la troublante ligne éditoriale des Cahiers bleus « Organe bimensuel de culture et d'organisation pour l'élaboration des doctrines du nouvel âge. Pour la création de l'État technique, coordonnateur des énergies pour la prospérité collective. Pour une nouvelle structure sociale propre à la société de producteurs excluant tout parasitisme. Pour une nouvelle culture fondée sur les nécessités du travail et sur la justice »

4 En 1934, lors de la parution du plus récent des deux ouvrages, les pouvoirs publics français venaient de confier la coordination rail-route à des comités techniques départementaux composés des représentants des deux filières. Fondés sur la concertation, leurs travaux n'aboutirent pas à des mesures concrètes. En 1936, le gouvernement $\mathrm{du}$ Front populaire voulut relancer l'affaire en adjoignant des représentants des "intérêts généraux » et des usagers auprès des professionnels du transport, sans plus de succès. La dernière tentative, imposée par le gouvernement Daladier en 1938, fut "autoritaire», et plus favorable à la route qu'au rail dont les déficits étaient statutairement couverts par le budget de l'État. Il était dès lors tentant pour ce dernier de pratiquer des coupes sombres de préférence dans le réseau ferré : de nombreuses lignes secondaires ont fermé en province; des services également; les tramways parisiens - déjà en net recul depuis dix ans - ont entièrement disparu dès avant la Seconde Guerre mondiale ; après, le repli fut quasi général. Dès lors la route et l'automobile connurent-elles un essor « sans entrave."

Le troisième ouvrage Chroniques routières. L'âge du pétrole 2007 déroule précisément cette histoire jusqu'à nos jours. CEuvre collective réalisée sous la supervision d'André Guillerme (qui a écrit l'introduction), elle est le catalogue de l'Exposition commémorant le centenaire du tout premier Congrès de la Route à Paris en 1908. L'association mondiale de la route (AIPCR) avait été fondée dans la foulée : elle fut un lieu de contacts et d'échanges d'expériences entre professionnels de tous bords (publics ou privés) mais aussi un lobby influent. L'ouvrage s'inscrit dans cette perspective mondiale. Il est divisé en de nombreux chapitres édités en version française, anglaise, espagnole. Les premiers chapitres abordent les temps forts de l'histoire de la route dans une perspective plus analytique qu'événementielle. "De la route avant l'automobile» chaotique, truffée d'ornières, mais vecteur d'échange et de pouvoir, à «la route de l'avenir » envers laquelle le consommateur multiplie les exigences, jusqu'à attendre de «son environnement immédiat un certain cachet », un "signe plus personnalisé », des embellissements susceptibles de lui conférer à terme une "valeur patrimoniale».

6 Suit un chapitre consacré " aux chiffres de la route». Son évolution centenaire est présentée sous forme de graphiques ainsi que dans un tableau synoptique regroupant 17 pays représentatifs. On y trouve l'évolution du nombre de véhicules à moteur, celui des kilomètres de routes et d'autoroutes construits, celui de la population totale des pays concernés. Des données plus détaillées sont également fournies pour la période récente, entre autres renseignements: la répartition des routes par catégories (autoroutes, nationales, secondaires, etc.), le pourcentage des portions revêtues. Comme dans le passé, le bilan est toujours contrasté entre les pays, y compris entre voisins à économie comparable. Ainsi la France et l'Allemagne font-elles à peu près jeu 
égal à l'aune des autoroutes, mais la première reste la championne toutes catégories au niveau des réseaux secondaires. Certaines données, de prime abord, paraissent étonnantes. En 1908, le linéaire de routes oscillait en moyenne entre trois et dix m par habitant, contre seulement $« 4,90 \mathrm{~m}$ en 2007 pour chaque terrien». La fameuse explosion routière tiendrait-elle dès lors du phantasme ? Évidemment non, nous disent les auteurs : en fait en cent ans, le linéaire de route a triplé mais en triplant également - et plus $(3,8)$ - l'évolution de la population mondiale en a atténué la perception. Enfin, « il y a route et route! ». Sur la période, le parc routier mondial est passé de moins de 500.000 véhicules à près d'un milliard, soit un facteur de croissance de l'ordre de 2000 . Ajoutons encore, dans les pas des auteurs, que le «linéaire n'est pas la capacité ». Les progrès techniques accumulés ont radicalement transformé la productivité de la route : ce n'est pas le même objet qu'on mesure d'un bout à l'autre de la période. Prudence donc dans la lecture des chiffres : «l'explosion de la mobilité » est donc bien là , avec d'ailleurs son cortège de grands chantiers routiers.

Les quinze chapitres suivants sont consacrés à ces chantiers, tout au moins à certains d'entre eux. Ensemble ils forment un panel étendu des travaux, pour la plupart spectaculaires, qui furent nécessaires au franchissement par la route de sites jusque-là improbables : ponts, tunnels, routes escarpées, routes en altitude, routes à la conquête de nouveaux territoires. Quinze monographies de chantiers choisis dans quinze pays de culture et de tradition différentes. Les textes sont agrémentés d'illustrations très fournies et d'une série graphique pour la période 1908-2007 rappelant les chiffres-clés du réseau du pays concerné. Le DVD-ROM de son côté nous montre en quinze vidéos " plan fixe » et en résumé, une journée entière de trafic en 2007, sur les 15 sites (vidéos présentées sur l'Exposition).

8 1908: année du Centenaire, mais aussi peut-être celle du basculement vers un autre paradigme où la route, l'automobile, ses usages, devront se réinventer! Nous en connaissons les causes : un pétrole plus rare et plus cher, les enjeux environnementaux. Dans un dernier chapitre, Hubert Peigné - coordonnateur du collège Infrastructures de transport au Conseil général des ponts et chaussées - nous propose une évaluation prospective des changements probables auxquels les hommes de l'art doivent s'attendre ... et se préparer.

\section{NOTES}

1. Don de François Sigaut. 
AUTEURS

DOMINIQUE LARROQUE

CDHTE - Cnam 